\section{Oxygen-cutting of Vanadium}

THE mechanism controlling the oxygen-cutting of iron and steel has been investigated by this Association, primarily along the lines of previous work by Wells ${ }^{2}$, whereby attention was focused on the iron/ oxygen combustion process. Recently this work has been extended to the study of the general conditions governing the progress of flame cuts through elementary materials, propagated by combustion with a pure gaseous element. The situation has been analysed in some detail for oxygen and chlorine. With the halogen gases, melting points of the elements most likely to be cut appear to be below the ignition temperature in the gas; no cuts were obtained in the cases of zinc and magnesium, which were otherwise thought to be likely to respond. Other halogen gases have not been tested experimentally, though it is known that fluorine may be used for flame-cutting copper ${ }^{2}$.

In the field of oxygen-cutting, probable natural flame-cutting elements are iron, titanium, vanadium and antimony. Experimentally, the latter element does not ignite below its melting point, but the first three are readily cut. Iron and steel cutting by oxyacetylene, hydrogen, propane, coal-gas or oxy-arc processes, has, of course, been in use for many years. Flane-cutting of titanium by the oxyacetylene process has formed the subject of a detailed report ${ }^{3}$, as it was not realized that the process was known in the United States ${ }^{4}$, though investigations by this Association were conducted independently.

Vanadium was selected as a trial element, since no previous tests were known, and, owing to the high cost of the material and its scarcity in massive form, it would be unlikely to be attempted outside a research laboratory. Because of the extremely poisonous nature of the vanadium oxides, it is necessary to conduct the cutting operation in a closed chamber, progress being viewed through a glass window. Two successful cuts have been made in a vanadium plate $11 \mathrm{~cm} . \times 5 \mathrm{~cm} . \times 0.6 \mathrm{~cm}$. obtained through the courtesy of the United Kingdom Atomic Energy Authority. The first cut was made manually and the second with a motor-driven oxyacetylene torch at $32 \mathrm{in} . / \mathrm{min}$. traverse speed. The surfaces near the pre-heat initiation point were contaminated with condensed vanadium metal and oxide, but away from the pre-heat area contamination was limited to a temper-coloured zone about $\frac{3}{8}$ in. on either side of the cut edge. There was no appreciable hardening on the surface of the plate to within $\frac{1}{32}$ in. of the cut. As in titanium, the major deleterious effect would be expected to be oxygen pick-up by the edge. Owing to the high reactivity of vanadium, its flame-cutting properties would be expected. to be more akin to titanium than to mild steel.

Apart from confirming previous conceptions of the process, the successful test on vanadium makes it likely that alloys of the iron-titanium-vanadium system would be 'cuttable' by oxygen methods.

A more detailed account of this work will appear elsewhere.

\section{G. Coates}

British Welding Research Association, Abington Hall, Cambridge.

'Wells, A. A., Brit. Welding J., 2, 392 (1955).

2 Priest, H. F., and Grosse, A. V., Indust and Eng. Chem., 39, 341 $(1947)$.

${ }^{3}$ Coates, G., Engineer, 208, 32 (1957).

"Everhart, J. L., "Titanium Alloys", 148 (Reinhold Pilot Books, New York, 1954).
Structure of Cupric Salts with Mono-, Di- and Tri-chloroacetic Acids

IN former communications ${ }^{1}$, we reported that the cupric salts, anhydrous or hydrated, with $n$ - and iso-monocarboxylic acids might consist of the dimeric molecules having a sort of copper-to-copper linkage. In continuation of these researches, we have recently examined the structure of cupric salts with mono-, di- and tri-chloroacetic acids, using the criteria previously derived: (1) the cupric alkanoates involving the dimeric molecules show, in addition to a band at about $43 \times 10^{13} \mathrm{c} / \mathrm{s}$., an absorption band of a special kind at about $80 \times 10^{13} \mathrm{c} . / \mathrm{s}$., which was regarded as due possibly to the presence of the copper-to-copper linkage; (2) the polarization of the latter band is the reverse of the polarization of the former.

The present measurements indicate that cupric monochloroacetate and dichloroacetate in dioxane show absorption spectra which are closely similar to those of the cupric alkanoates in solution, showing an absorption band of the special kind at about $80 \times 10^{13} \mathrm{c} . / \mathrm{s}$. The dichroism in the visible and ultra-violet of the two compounds has been determined quantitatively by Tsuchida-Kobayashi's microscopic method ${ }^{2}$. The crystals of cupric monochloroacetate tetrahydrate and anhydrate, and of anhydrous cupric dichloroacetate all show absorption bands at about 41 and $80 \times 10^{13} \mathrm{c} . / \mathrm{s}$. besides strong absorption in the shorter wave-length regions. The polarization of the band at about $80 \times 10^{13} \mathrm{c} . / \mathrm{s}$. is found to be the reverse of the polarization of the band at about $41 \times 10^{13} \mathrm{c} / \mathrm{s}$. These relationships bear a close resemblance to those with the cupric alkanoates, suggesting the existence of dimeric molecules similar to those of the alkanoates. Thus it may be concluded that cupric monochloroacetate and dichloroacetate have the dimeric molecules in dioxane and in the crystalline state. The dimeric molecules may be represented by $\mathrm{Cu}(R \cdot \mathrm{COO})_{4}$ or $\mathrm{Cu}(R \cdot \mathrm{COO})_{4} \cdot\left(\mathrm{OH}_{2}\right)_{2}$.

In contrast to the above mentioned compounds, cupric trichloroacetate in dioxane shows only one absorption band at about $40 \times 10^{13} \mathrm{c} / \mathrm{s}$. in the corresponding wave-length range. Furthermore, cupric trichloroacetate tetrahydrate in the crystalline state displays polarized absorption spectra having only the absorption band at about $40 \times 10^{13} \mathrm{c} . / \mathrm{s}$. Thus the spectra of the compound are found to be quite different from those of the other two compounds. It is concluded, therefore, that the trichloroacetate does not involve the dimeric molecules in the crystalline state or in dioxane.

Cupric dichloroacetate is known to exist in a tetrahydrate as well. The dichroism of this form is not quite identical with that of the anhydrous form, which is shown to consist of the dimeric molecules. The structure of the tetrahydrate in the crystalline state is under investigation.

\section{Ryutaro Tsuchida} ShoIChIRo YAMADA Hrroji Nakamura

Department of Chemistry, Faculty of Science,

Osaka University, Japan.

Nov. 7.

1 Tsuchida, R., and Yamada, S., Nature, 176, 1171 (1955). Tsuchida, R., Yamada, s., and Nakamura, H.,' "Truchida, R., and Kobayashi, M., Bull. Chem. Soc. Japan, 13, 619 (Osaka, 1944). 